\title{
Digitale Inklusion: Zur sozialen Öffnung des Wissenschaftssystems Digital Inclusion: The Social Implications of Open Science
}

\author{
Sascha Dickel \\ Friedrich Schiedel-Stiftungslehrstuhl für Wissenschaftssoziologie an der Technischen Universität München, \\ Arcisstraße 21, 80333 München, Germany. \\ sascha.dickel@tum.de

\section{Martina Franzen} \\ Wissenschaftszentrum Berlin für Sozialforschung, Forschungsgruppe Wissenschaftspolitik, Reichpietschufer 50, \\ 10785 Berlin, Germany. \\ martina.franzen@wzb.eu
}

\begin{abstract}
Zusammenfassung: Aus dem Blickwinkel der Systemtheorie gilt die Wissenschaft prototypisch als ein selbstreferentielles Funktionssystem, das eine soziale Distanz zur Öffentlichkeit unterhält. In der funktional differenzierten Gesellschaft ist die Inklusionsordnung der Wissenschaft strikt an verberuflichte Leistungsrollen gebunden, während alle übrigen Gesellschaftsmitglieder weitgehend exkludiert werden. Unsere These ist, dass im Zuge des digitalen Wandels neue Inklusionsmuster entstehen. Wir beobachten die Verbreitung funktionalisierter Subrollen, in denen die professionelle Rolle des Wissenschaftlers dekomponiert wird. Eine gesellschaftstheoretisch informierte Beschreibung dieser neuen digitalen Inklusionsprofile leistet zweierlei: Zum einen wird dadurch ein differenzierungstheoretischer Konservatismus überwunden, der Diagnosen einer Öffnung der Wissenschaft lediglich als semantische Oberflächenphänomene deutet. Zum anderen lässt sich analytische Distanz zu dominanten Selbst- und Fremdbeschreibungen gewinnen, die diese Entwicklung als Demokratisierung der Wissenschaft rhetorisch überhöhen.
\end{abstract}

Schlagworte: Wissenschaftssoziologie; Inklusion; Citizen Science; Web 2.0; gesellschaftliche Differenzierung.

Summary: From the perspective of systems theory, science is a prototype of a self-referential functional system that maintains social distance to the public. In functionally differentiated societies, science maintains a strict regime of inclusion, which is closely tied to the professional role of the scientist as someone who produces and acquires knowledge. We suggest that the digital revolution is generating novel modes of inclusion. These take the form of functionalized subroles in which the professional role of the scientist is disassembled. By proposing a socio-theoretically informed characterization of these new modes of inclusion we aim to meet two different goals: The first is to overcome the theoretical conservatism of differentiation theory, in which diagnoses of the social openness of science are solely interpreted as semantic surface phenomena. The second is to achieve analytical distance to a societal discourse that describes these new modes of inclusion as examples of a successful democratization of science.

Keywords: Sociology of Science; Inclusion: Citizen Science; Web 2.0; Societal Differentiation.

\section{Einleitung: Geschlossenheit und Offenheit der Wissenschaft}

Die Wissenschaft etablierte sich in der modernen Gesellschaft als eine distinkte Form sozialer Praxis, die in der Professionalisierung erfahrungswissenschaftlichen Handelns (Oevermann 2005) sowie der Genese spezifisch wissenschaftlicher Organisationen (Whitley 2010) und Fachgemeinschaften (Gläser 2006) ihre konkreten sozialen Formen fand. Insbesondere die differenzierungstheoretisch geprägte Gesellschaftstheorie betont die selbstreferentielle Schließung des wissenschaftlichen Kommuni- kationszusammenhangs, die sich im Verlauf des 20. Jahrhunderts endgültig verfestigte (Luhmann 1990; vgl. auch Schimank 2012). Diese Beobachtung einer zunehmenden Geschlossenheit steht jedoch im Kontrast zu Beobachtungen der Science and Technology Studies (STS), die seit Jahrzehnten (vgl. nur Gibbons et al. 1994) fast unisono auf eine zunehmende Öffnung der Wissenschaft zur Gesellschaft aufmerksam machen.

Der vorliegende Beitrag schlägt daher eine Brücke zwischen den Diagnosen der STS und der soziologischen Differenzierungstheorie - allerdings nicht im Sinne einer kaum zu leistenden theoretischen Inte- 
gration, sondern durch eine gesellschaftstheoretische Re-Interpretation der neueren Öffnungstendenzen der Wissenschaft. Zentral für unsere Überlegungen ist dabei der Begriff der Inklusion, unter dem in differenzierungstheoretischer Perspektive die Frage behandelt wird, wer unter welchen Bedingungen an funktionssystemischer Kommunikation beteiligt ist. Grundlegend für die Inklusionsordnung der modernen Wissenschaft ist die Einrichtung einer Berufsrolle (des Wissenschaftlers), die für die systemeigene Leistungsproduktion verantwortlich ist, während alle übrigen Gesellschaftsmitglieder aus der wissenschaftlichen Wissensproduktion weitgehend exkludiert werden (Stichweh 1988a; Ben-David 1991).

Unsere These ist, dass sich die rezente Öffnung der Wissenschaft zur Gesellschaft als Transformation dieser strikten Inklusionsordnung im Zuge medialer Wandlungsprozesse interpretieren lässt. Insbesondere die Digitalisierung ermöglicht neue funktionale Beziehungen zwischen Wissenschaftlern und Nicht-Wissenschaftlern. Eine gesellschaftstheoretisch adäquate Beschreibung dieser Beziehungen leistet zweierlei: Zum einen wird dadurch ein differenzierungstheoretischer Konservatismus überwunden, der Diagnosen einer Öffnung der Wissenschaft lediglich als semantische Oberflächenphänomene deutet, zum anderen lässt sich damit eine soziologische Distanz zu Beschreibungen in STS und Wissenschaftspolitik gewinnen, welche diese Entwicklung als Demokratisierung der Wissenschaft rhetorisch überhöhen.

In einem ersten Schritt rekapitulieren wir die differenzierungstheoretische Diskussion zur sozialen Inklusion im Allgemeinen und zur Inklusionsordnung der Wissenschaft im Besonderen (2). Dabei greifen wir auch und gerade Beiträge aus den STS zur Öffnung der Wissenschaft auf (3). Danach widmen wir uns neuen Inklusionsmustern vor dem Hintergrund des digitalen Wandels und entwerfen eine Typologie rezenter nicht-professioneller Rollen in der Wissensproduktion (4). Im Fazit arbeiten wir schließlich die Konsequenzen der sozialen Öffnung der Wissenschaft heraus: Durch Digitalisierung erscheint eine dezentrierte Wissensproduktion vorstellbar, die in der Sozialdimension nicht mehr primär durch die Berufsrolle des Wissenschaftlers strukturiert ist, der verschiedene wissenschaftliche Funktionen in sich bündelt und qua professioneller Autorität monopolisiert (5).

\section{Inklusion im Wandel}

In differenzierungstheoretischer Perspektive werden die Geschlossenheit und Offenheit gesellschaftlicher Funktionsbereiche in der Sozialdimension mit dem Begriff der Inklusion erfasst. In einem programmatischen Aufsatz aus dem Jahr 1988 entfaltet Stichweh im Anschluss an Niklas Luhmann die These funktionssystemspezifischer Inklusionsordnungen. Fast alle Funktionssysteme der modernen Gesellschaft sind demzufolge durch einen doppelten Inklusionsmodus geprägt, der in einer Differenz von Leistungs- und Publikumsrollen zum Ausdruck kommt. Während Leistungsrollen für die teilsystemische Leistungsproduktion zuständig sind, sorgen Publikumsrollen für die Inklusion der Gesamtbevölkerung in das jeweilige Sozialsystem und damit in die Gesellschaft (Stichweh 1988b: 261). Leistungs- und Publikumsrollen stehen demnach komplementär zueinander. Dies lässt sich am einfachsten am Funktionssystem Politik veranschaulichen, dessen Leistungsrollenträger Politiker sind, während die Wähler die Rolle des Publikums einnehmen. Für den Sport lässt sich analog auf den Leistungssportler und den Zuschauer verweisen, für die Medizin auf den Arzt und den Patienten (vgl. eine Gesamtdarstellung bei Burzan et al. 2008: 32). Die soziale Schließung von Funktionssystemen erfolgt somit auf der Ebene der Leistungsrollen, ihre soziale Öffnung wird hingegen durch die universelle Figur einer komplementären Publikumsrolle hergestellt. Die Figur des Laien wird damit zum einen generalisiert, da prinzipiell jeder eine Publikumsrolle einnehmen kann, zum anderen spezifiziert, da der Laienstatus in jedem Funktionssystem anders bestimmt ist und sich unmittelbar auf die Leistungsrolle bezieht (Stichweh 1988b: 262). Mit diesem Begriff des Publikums ist somit nicht die Gesellschaft, sondern eine jeweils funktionssystembezogene Rolle bezeichnet. Der moderne Leistungssport ist ohne Zuschauer ebenso undenkbar wie das Medizinsystem ohne Patienten. Beide Rollentypen sind also in ihrer Komplementarität systemkonstitutiv.

Um die scharfe Asymmetrisierung von Leistungsund Publikumsrollen auszutarieren, haben sich laut Stichweh ausgleichende Mechanismen entwickelt, allen voran die Ausbildung sekundärer Leistungsrollen, die er als „aktivistische [...] Alternative zu einem reinen Publikumsstatus“ begreift. $^{1} \mathrm{Zu}$ ihnen

\footnotetext{
${ }^{1}$ Volkmann weist darauf hin, dass sekundäre Leistungsrollen auch dahingehend typisiert werden können, ob sie sich als Alternative oder als Zuarbeiter für primäre Leistungsrollen positionieren (Volkmann 2010: 216f.).
} 
gehören sowohl Amateur- als auch Kritikerrollen. Die Herausbildung sekundärer Leistungsrollen wird in dem Umstand verortet, dass in der Moderne die Partizipation an Handlungszusammenhängen (Leistungsrolle) gegenüber der Beobachtung von Handlungszusammenhängen (Publikumsrolle) eine höhere Wertschätzung erfährt (Stichweh 1988b: 280).

Eben diesem Partizipationsimperativ folgend hat sich die asymmetrische Inklusionsordnung der modernen Gesellschaft seit den 1960er Jahren stetig relativiert. Am augenfälligsten ist dies gewiss in der politischen Sphäre im Kontext sozialer Bewegungen $\mathrm{zu}$ beobachten. Doch auch in anderen Gesellschaftsbereichen werden immer weiterreichendere Partizipationsansprüche formuliert, die Gerhards (2001) allgemein als „Aufstand des Publikums“ beschreibt. Sie finden ihre institutionelle Gestalt in der Gründung von Organisationen und Netzwerken wie Patientengruppen (Medizin) und Verbraucherschutzorganisationen (Wirtschaft). Eine wichtige Rolle beim Aufstand des Publikums spielen die Massenmedien, die Spezialwissen allgemein zugänglich und zugleich öffentlich kritisierbar machen (Pfadenhauer 2003: 177f.).

Eine darüber hinausgehende Steigerung von Publikumsaktivitäten - verbunden mit einem Ausbau sekundärer Leistungsrollen - wird mit dem Aufkommen des Internets und insbesondere mit dem „Web $2.0^{\text {“2 }}$ diagnostiziert, dem im besonderen Maße ein Potenzial für neue Möglichkeiten der Interaktion, Kollaboration und Teilhabe zugesprochen wird (Schrape 2010). In ökonomischer Lesart werden diese Tendenzen als Aufstieg der Prosumenten ${ }^{3}$ gedeutet, Konsumenten also, die sich aus der Passivität der Konsumentenrolle lösen und selbst zu (Co-)Produzenten werden - ohne dabei notwendigerweise den „Umweg“ über organisationale Strukturen gehen zu müssen. Entsprechend ist hier von einer "democratization of production“ (Shirky 2008: 297) durch Prosumtion im Web 2.0 die Rede. Volkmann (2010) weist darauf hin, dass sich das eher unscharfe Konzept des Prosumenten differenzierungstheoretisch im Sinne des Begriffs der sekundären Leistungsrolle universalisieren und theoretisch präzisieren lässt.

\footnotetext{
${ }^{2}$ Der Kompaktbegriff des Web 2.0 bezieht sich auf die Ausbreitung sozial-medialer Infrastrukturen, die Nutzern die Möglichkeit geben, miteinander in Austausch zu treten und User Generated Content zu produzieren.

${ }^{3}$ Zur aktuellen Diskussion vgl. Blättel-Mink \& Hellmann 2010. Zur ursprünglichen Bedeutung vgl. Toffler 1980.
}

In vielfältigen sozialen Kontexten sind Personen gegenwärtig als sekundäre Leistungsrollenträger im Netz aktiv, sei es als Jedermann-Journalist (Volkmann 2010; Reimer \& Rupper 2012), als Hobbymusiker oder Freizeitdesigner. Aktive Inklusionsprofile sind, so scheint es, auch jenseits von Leistungsrollenträgern in der Moderne mittlerweile eine gesellschaftliche Normalität. Der digitale Wandel schafft neue Gelegenheitsstrukturen für die individuelle Partizipation an gesellschaftlichen Handlungszusammenhängen.

\section{Inklusion in der Wissenschaft - ein Sonderfall}

Folgt man der systemtheoretischen Einordnung, gestaltet sich Inklusion im Funktionssystem Wissenschaft grundlegend anders als in den übrigen Funktionsbereichen. Im Hinblick auf die Ausbildung von Publikums- und sekundären Leistungsrollen erscheint die Wissenschaft als Sonderfall. So wird hier keine Asymmetrie zwischen einer verberuflichten Leistungsrolle und einer spezifischen Publikumsrolle angenommen: Es sind vielmehr Wissenschaftler, die in Personalunion sowohl entsprechende Leistungen erbringen als auch an diesen partizipieren. Ein spezifisch wissenschaftliches $\mathrm{Pu}$ blikum, dem - wie bei anderen Systemen - eine systemkonstitutive Rolle zukäme, ist in der modernen Wissenschaft nicht auszumachen. Stattdessen fallen Produktion von Leistung (Wissen) und Leistungsabnahme (Rezeption) in der Rolle des Berufswissenschaftlers zusammen.

Die moderne Wissenschaft bildet somit geradezu den Idealtyp eines selbstreferentiellen Kommunikationszusammenhangs. Im 17. und 18. Jahrhundert war die höfische Gesellschaft noch der erste Adressat der Wissenschaft, nicht zuletzt weil die um Glaubwürdigkeit und Anerkennung ringenden $\mathrm{Na}$ turforscher noch auf den sozialen Rang der bezeugenden Zuschauer in öffentlichen Demonstrationen angewiesen waren (Shapin \& Schaffer 1985). Dies ändert sich mit der Umstellung auf schriftliche Kommunikation ${ }^{4}$ im Rahmen periodisch erscheinender Fachzeitschriften, die sich zunehmend spezialisieren und damit gleichzeitig der Binnendifferenzierung der Wissenschaft in Disziplinen und Subdisziplinen Rechnung tragen. Ein wesentliches

\footnotetext{
4 „Damit verlagert sich das Problem, andere zu überzeugen, von der unmittelbaren Demonstration von Evidenz auf die mittelbare Darstellung in Texten." (Weingart 2005: 15)
} 
Kennzeichen wissenschaftlicher Texte sind präzise definierte Fachtermini, die den Idealen der Exaktheit, Kontextunabhängigkeit, Eindeutigkeit und evaluativen Neutralität von Wissenschaft entsprechen (Auer \& Baßler 2007: 13 ff.). Diese für Laien weitgehend unverständliche Sprache sowie eine auf häufig nicht-explizierten Annahmen aufbauende Argumentation machen eine "drastische Reduktion von verstehensfähigen Adressaten unvermeidlich“ (Luhmann 1990: 624). Popularisierung ${ }^{5}$ wird seit Beginn des 20. Jahrhunderts wissenschaftsintern als inhaltliche Verzerrung wahrgenommen und verliert dadurch ihre Akzeptanz (Weingart 2005: 19).

Eine publikumsbefreite Wissenschaft ist offenbar das Resultat einer Professionalisierung von Forschung, die eine Deinstitutionalisierung von bis zu Beginn des 19. Jahrhundert verbreiteten Inklusionsformen beinhaltete und vermutlich gerade aufgrund ihrer präskriptiven Autonomie so erfolgreich expandierte (Merton 1942). Für die moderne Wissenschaft erscheint daher die sekundäre Leistungsrolle als eine mittlerweile zu vernachlässigende Größe (Burzan et al. 2008; Schimank 2012). Historisch betrachtet gilt das 18. Jahrhundert als "goldenes Zeitalter des Amateurforschers" (Hochadel 2003: 41), das die institutionelle Trennung zwischen Laien und Experten so noch nicht kennt. Die Beiträge von Amateurwissenschaftlern, welche noch im 19. Jahrhundert als Ausdruck der Emanzipationsansprüche eines aufstrebenden Bürgertums galten, werden im 20. Jahrhundert von der professionellen Wissenschaft jedoch zunehmend ignoriert. ${ }^{6}$ Während Amateurforscher auch unter den Bedingungen einer beginnenden wissenschaftlichen Professionalisierung noch bis ins frühe 20. Jahrhundert hinein eine gewisse Rolle spielten (Mahr 2014), hat sich die Exklusion der Amateure in der Wissenschaft im 20. Jahrhundert offenbar doch in den meisten Disziplinen durchgesetzt.

„Es gibt zwar [...] die sekundäre Leistungsrolle des Amateurwissenschaftlers, etwa des Lokalhistorikers, der in seiner Freizeit die Geschichte der Heimatstadt während der industriellen Revolution aufarbeitet, oder des Käfersammlers, der die lokale Artenvielfalt dokumentiert. Doch nur die wenigsten Gesellschaftsmitglieder nehmen heutzutage

\footnotetext{
${ }^{5}$ Im Französischen wird mit dem Begriff der „vulgarization" die negative Konnotation von Popularisierung umso deutlicher (Bensaude-Vincent 2001).

${ }^{6}$ Stichweh (1988a: 98f.) nennt in diesem Zusammenhang vor allem die Beweisvorschläge von Amateuren zu klassischen mathematischen Problemen, die seitens der Akademien und Wissenschaftsverwaltungen ab Ende des 18. Jahrhunderts nicht mehr geprüft wurden, was auf Exklusionseffekte hinweist.
}

noch auf diese Weise an wissenschaftlicher Forschung teil; und ihr Beitrag zum Erkenntnisfortschritt, der in den ersten Jahrhunderten der modernen Wissenschaft durchaus nicht zu vernachlässigen war, hat sich nahezu auf Null reduziert." (Schimank 2012: 120f.; ähnlich bereits Stichweh 1988b: 282f.)

Ähnliches gilt für die Rolle des Kritikers, die in der Wissenschaft so nicht existiert. Während die Kunst spätestens seit der Romantik den professionellen Kritiker „als Sprachrohr des interessierten Laien“ kennt (Grohs 1964), wird diese Rolle in der Wissenschaft ebenfalls von professionellen Wissenschaftlern ausgefüllt, wie es bereits im Begriff des „Peer Review“ zum Ausdruck kommt. Wenn die Aufgabe des Kritikers allgemein darin besteht, zwischen dem Leistungsrollenträger und dem Publikum zu vermitteln, dann schafft alleine schon die wissenschaftliche Fachterminologie eine Grenze, die sich jeder Allgemeinverständlichkeit entzieht und dadurch gegen nicht-wissenschaftliche Formen öffentlicher Kritik immunisiert.

Bezeichnenderweise spielt die Wissenschaft sowohl in Gerhards Ausführungen zur Aktivierung des Publikums als auch in neueren Arbeiten zum Prosumenten keine Rolle. In der Tat schien sich die wissenschaftliche Wissensproduktion einer sozialen Öffnung lange Zeit zu entziehen - und dies deckt sich mit der theoretischen Annahme einer fehlenden spezifischen Publikumsrolle, an der sich Partizipationserwartungen kristallisieren könnten. Eine theoretische Erklärung dieses Sonderfalls mag in der eigentümlichen Struktur der Wissenschaft und ihrer Kerninstitution Universität liegen, Forschung und Lehre zu verknüpfen und damit ihr Publikum im Außen, nämlich im Erziehungssystem zu suchen. Wissenschaftler sehen sich, sofern sie an einer Hochschuleinrichtung arbeiten, mit einer dualen Funktion ausgestattet, Forscher und Lehrer zugleich zu sein. Der Vollzug gesamtgesellschaftlicher Inklusion verläuft somit indirekt in Form der Hochschulerziehung, ohne für den Bereich der Forschung eine analoge Nachfrage zu generieren (Stichweh 1988b: 275f.).

Statt der Ausbildung eines spezifischen und konstitutiven Publikums gewinnt in Zeiten von „Medialisierung " (Weingart 2012) demgegenüber die medienvermittelte Inklusion eines generalisierten Publikums in das Wissenschaftssystem an Stärke. Wissenschaft ist mehr als je zuvor in den Medien präsent. Seit Beginn der 1990er Jahre ist der quantitative Anteil der Wissenschaftsberichterstattung gestiegen und hat jüngst ein historisch einmaliges Niveau erreicht (Bauer 2012). Zeitgleich lässt sich von einer Professionalisierung des Wissenschafts- 
journalismus sprechen. Berufsverbände (wie in Deutschland die Wissenschaftspressekonferenz) wurden gegründet, Studiengänge zum Wissenschaftsjournalismus eingerichtet und professionsethische Normen begründet, die nur für diesen journalistischen Bereich Geltung beanspruchen.

Mit dem allgemeinen Wissenschaftsboom institutionalisierten sich in Form von Public Relations Grenzstellen zwischen wissenschaftlichen Forschungseinrichtungen und den Medien als genuine „boundary organizations“ (Guston 2006) wie das britische Science Media Centre (Fox 2012). Formate, die einen interaktiven Bezug zur Öffentlichkeit herstellen, z.B. Kinderuniversitäten, Science Center oder jährliche lokale Veranstaltungen wie die „Lange Nacht der Wissenschaften“ haben sich vervielfältigt (Bucchi \& Trench 2014).

Auf der Basis einer vergleichenden Untersuchung von Inklusionsprofilen in allen Teilsystemen zeigt die empirische Studie von Burzan et al. (2008) gleichwohl, dass die Wissenschaft (zusammen mit dem Recht) die schwächste Inklusionsintensität aufweist: „Kaum jemand ist als Amateurforscher tätig, noch immer nicht allzu viele interessieren sich - als medienvermittelte indirekte Inklusion - für die Wissenschaftsseiten der Zeitungen und Zeitschriften sowie für Wissenschaftssendungen im Fernsehen. Die Wissenschaft betrifft uns zwar massiv, aber über das Wirken anderer Teilsysteme und in anderen Inklusionsverhältnissen.“ (Burzan et al. 2008: 103f.)

Die Exklusion der Laien aus der Wissenschaft wird unter politischen Gesichtspunkten seit dem späten 20. Jahrhundert zunehmend kritisiert. Wissenschaftskommunikationsaktivitäten sollten die entstandene Kluft von Wissenschaft und Gesellschaft $\mathrm{zu}$ überwinden helfen, die sich mit den öffentlich ausgetragenen Debatten um die wissenschaftlichtechnische Risikoproduktion wie im Falle der Kernenergie oder Gentechnik stetig vergrößert hat. Ausgehend von Großbritannien werden in den 1980er Jahren Programme aufgelegt, die als „Public Understanding of Science“ zunächst dem traditionellen Popularisierungsparadigma folgen. Unter den Bedingungen der wachsenden Legitimationskrise wissenschaftlicher Experten wird das lineare Modell ab Mitte der 1990er Jahre zugunsten des Dialogmodells „Public Engagement with Science and Technology“ aufgegeben (Bauer et al. 2007).

„Wissenschaft im Dialog“" 7 lautet der programmatische Ausweg, um Interaktionsmöglichkeiten zu

\footnotetext{
${ }^{7}$ Dies ist auch zugleich der Name der Initiative für Wissenschaftskommunikation in Deutschland, kurz WiD,
}

erhöhen und Akzeptanzprobleme zu überwinden. Der „uninvited participation“, welche die Form allgemeiner oder spezifischer Wissenschafts- und Technikkritik bzw. politischer Interventionen von zivilgesellschaftlichen Akteuren artikulierte, wird mit Modellen einer „invited participation“ zu begegnen versucht, die auf selektive Inklusion von Bürgern in strukturierten deliberativen Arrangements setzen (Wynne 2007; Wehling 2012).

Im Zuge vielfältiger Problematisierungen wissenschaftlicher Expertise - auch und gerade durch die Sozialwissenschaften - wird die Exklusivität wissenschaftlicher Wissensproduktion zunehmend infrage gestellt und zugleich eine aktivere Partizipation der Laien - zumindest in bestimmten Forschungskontexten ${ }^{8}$ - als systemrelevantes Erfordernis zur Produktion sozial robusten Wissens markiert (Wynne 1992; Epstein 1995; Brown 2007). Programmatiken einer Demokratisierung der Wissenschaft (Kitcher 2011), die durch Konzepte wie „post-normal science“ (Funtowicz \& Ravetz 1993) oder „mode 2“ (Gibbons et al. 1994) ihren gesellschaftsreflexiven Ausdruck finden, diagnostizieren und fordern daher eine zunehmende Berücksichtigung nicht-zertifizierten Laienwissens ${ }^{9}$ in der wissenschaftlichen Wissensproduktion. "The context speaks back" lauten Diagnose und Diktum zugleich (Nowotny et al. 2001). ${ }^{10}$ Jeder zweite Deutsche wünscht sich heute offenbar mehr Beteiligungsmöglichkeiten an Entscheidungen über Wissenschaft und Forschung, so das Ergebnis einer aktuellen repräsentativen Umfrage (Wissenschaftsbarometer 2014).

Festzuhalten bleibt, dass das Publikum, das über Public Engagement bzw. Wissenschaftskommunikationsaktivitäten erreicht und vermutlich stetig

vom Stifterverband 1999 gegründet und gefördert von den zentralen Wissenschaftsorganisationen.

${ }^{8}$ Insbesondere solchen, die im besonderen Maße mit Risiken und Unsicherheiten assoziiert werden (Funtowicz \& Ravetz 1993) und von denen Mensch und Natur negativ betroffen sein können (Irwin 1995).

${ }^{9} \mathrm{Im}$ Bereich der zivilgesellschaftlichen Partizipation an Wissenschaft gerät der Begriff des Laien dann an seine Grenzen, wenn Verwissenschaftlichungsprozesse einsetzen und die kritische Distanz zur dominanten Expertenmeinung verloren geht (Epstein 2011).

${ }^{10}$ Die postulierte Symmetriebeziehung zwischen Wissenschaftlern und Bürgern lässt sich in der Praxis kaum durchhalten. Empirische Untersuchungen weisen darauf hin, dass sich in der Laienpartizipation im Kontext deliberativer Politik die institutionell abgesicherte Experten/Laien-Differenz subkutan reproduziert (vgl. etwa Junge 2008; Bogner 2010; Bora 2010). 
vergrößert wird, gleichwohl immer noch keines ist, an dem sich eine genuine Publikumsrolle im strengen Sinne der Stichweh'schen Definition ausbilden kann. Während der Wähler für die Politik oder der Konsument für die Wirtschaft konstitutiv und für die Kontrolle der Leistungsabgaben zuständig sind, bleibt das angesprochene Publikum der Wissenschaftskommunikation quasi außen vor. Weder im Modus Bildung noch im Modus Medienrezeption qua Populärinklusion lässt sich sinnvollerweise von einer systemspezifischen Publikumsrolle sprechen. Beide Formen ${ }^{11}$ indirekter Inklusion verweisen auf ein universelles Publikum, das jedoch nicht respezifiziert ist und somit keine systemdefinierende Funktion übernehmen kann. Vielmehr sind diese beiden vermittelten Inklusionsmechanismen in allen Teilsystemen wirksam. Der Unterschied besteht dann einzig in der Größe des jeweiligen Publikums, das auf diese Weise inkludiert wird (vgl. Burzan et al. 2008). Deliberative Arrangements wiederum verweisen ebenfalls nicht auf die Ausbildung einer wissenschaftsspezifischen Laienrolle, sondern sind vornehmlich als wissenschaftspolitische Verfahren zu betrachten (Abels \& Bora 2004; Bora 2005).

Es existieren in der Wissenschaft demnach weder institutionalisierte Formen einer Rückäußerung des Publikums noch einer Beobachtung der Publikumsreaktionen durch die Wissenschaft und somit keine Formen einer direkten Inklusion, die systemkonstitutiv wären (Stichweh 1988b; vgl. auch Burzan et al. 2008: 38). Diese theoretische Sichtweise ist jedoch nicht nur normativ umstritten (Collins \& Evans 2002; Jasanoff 2011), sondern scheint vor allem angesichts der Emergenz neuer Kommunikationstechnologien brüchig geworden zu sein.

\section{Neue wissenschaftliche Inklusionsmuster im digitalen Wandel}

Digitale Medien verändern die wissenschaftliche Kommunikation. Die Rede ist heute von einer zweiten wissenschaftlichen Revolution, die sich analog zur ersten wissenschaftlichen Revolution zunächst als Neuerung im Bereich der Publikationsformen niederschlägt. Mit der Umstellung vom Buchdruck auf elektronische Verbreitungstechnologie und der

\footnotetext{
11 Für den Modus Bildung ist allerdings erneut darauf hinzuweisen, dass das genuine Publikum der Wissenschaft im Erziehungssystem verortet wird (Stichweh 1988b), das über die Organisation Universität und der sie tragenden Idee der Einheit von Forschung und Lehre mit der Wissenschaft gekoppelt ist.
}

Bewegung hin zu Open Access befindet sich die interne Wissenschaftskommunikation seit den 1980er Jahren in einer Umbruchphase (Franzen 2011a: 82ff.). Im Falle von Open Access-Publikationen können Autoren prinzipiell jeden erreichen, da institutionelle Zugriffsbarrieren wegfallen. Gleichzeitig verändern sich im digitalen Raum die Rezeptionsgewohnheiten und die Bewertungsmöglichkeiten werden erweitert. Im Folgenden werden wir zeigen, dass digitale Medien die Voraussetzungen für eine Generalisierung des Publikums der Wissenschaft schaffen. Darüber hinaus bieten sie für Nicht-Wissenschaftler Opportunitätsstrukturen, um am Forschungshandeln partizipieren zu können. Durch die politische Programmatik der Demokratisierung der Wissenschaft werden diese Öffnungstendenzen befördert mit dem Ziel, die Effizienz der Forschung zu erhöhen und in ihren Wirkungen gesamtgesellschaftlich zu legitimieren. In der Gesamtschau werden damit Konturen neuer Inklusionsprofile sichtbar, welche die professionelle Schließung des Wissenschaftssystems relativieren.

\subsection{Ein neues Publikum}

Eine Vorreiterrolle in dem Prozess der programmatischen Publikumserweiterung wissenschaftlicher Kommunikation hat die „Public Library of Science“ (PLOS) übernommen, die als Open Access Platform 2001 gegründet wurde und von Beginn an das Ziel verfolgte, die wissenschaftsinteressierte Öffentlichkeit in ihr Publikationsangebot miteinzubeziehen: „PLOS believes that the general public should have access to the scientific literature, tools to understand that literature, and even the opportunity to engage in scientific debates." (Gross 2012) Um die inhaltliche Zugänglichkeit wissenschaftlicher Artikel zu erhöhen, werden in den PLOS-Fachzeitschriften wie PLOS Medicine, PLOS Biology oder dem multidisziplinären Journal PLOS ONE sogenannte Synopsen begleitend zu ausgewählten Artikeln veröffentlicht, die der Kontextualisierung und damit der allgemeinen Zugänglichkeit neuer Forschungsergebnisse dienen. Hinzu gesellen sich Multimedia-Elemente wie Blogs, Videos oder Podcasts, die inzwischen zahlreiche Zeitschriften anbieten, die, gekoppelt an soziale Medien wie Twitter, YouTube oder Facebook, Informationen an ein breites Publikum vermitteln, ohne den Umweg über die klassischen Massenmedien zu nehmen. ${ }^{12}$ Neben den Informalisierungstendenzen innerhalb der wis-

\footnotetext{
12 Das Facebook Profil von Nature erreicht laut Selbstdarstellung über 6 Millionen Besucher pro Monat.
} 
senschaftseigenen Medien und dem Wissenschaftsboom in den Massenmedien hat sich auch das öffentliche Beobachtungsspektrum von Wissenschaft vervielfältigt. Im Zuge der Umstellung auf elektronisches Publizieren sind Plattformen entwickelt worden, die Bewertungen bereits erschienener Artikel erlauben („post publication“ statt „pre-publication peer review“). Jenseits der Massenmedien existieren gegenwärtig zahlreiche spezielle Science Blogs, die über wissenschaftliche Neuerscheinungen im (natur-)wissenschaftlichen Bereich berichten und diese evaluieren (z.B. Research Blogging) oder auf Verfehlungen im Publikationswesen aufmerksam machen (z.B. Retraction Watch). Darüber hinaus stehen Wikis zur kollaborativen Plagiatssuche und -dokumentation wie GuttenPlag prinzipiell jedermann zur Mitarbeit offen. Im Buchbereich ist das Unternehmen Amazon zum größten Literaturkritik-Portal avanciert - auch für Sach- und Fachbücher. Zu der klassischen Gutachterrolle gesellen sich somit neue Formen der öffentlichen (Laien-)Kritik (Nentwich \& König 2012; Franzen 2015a i.E.).

Dies kommt der normativen Forderung eines „extended peer review " schon sehr nahe (Funtowicz \& Ravetz 1993). Neben elektronischen Kommentarfunktionen ${ }^{13}$, die zahlreiche wissenschaftliche Zeitschriften registrierten Nutzern inzwischen offerieren, hat PLOS die sogenannten Article-Level Metrics eingeführt. Dahinter steht die Idee, den Impact eines Beitrags nicht mehr allein über Zitationen zu messen, sondern über seine gesellschaftliche Resonanz (PLoS Medicine Editors 2006). Als Resonanzfaktoren werden neben Zitationen weitere Daten wie Medienberichte, Tweets, Bookmarkings oder Downloads zu einem Artikel ausgewertet (Lin \& Fenner 2013).

Auf Basis dieser sogenannten Altmetrics (Priem 2013) - alternativ zu reinen Zitationszahlen - werden seit 2009 Software-Tools entwickelt, um den Impact eines singulären Artikels über nutzergenerierte Daten im Web 2.0 zu erfassen. Der verbreitetste Indikator ist der vom gleichnamigen Unternehmen entwickelte Altmetric Score, der sich als besonderes Feature in jedes Verlagsangebot integrieren lässt. In den Algorithmus des Altmetric Score fließen keine herkömmlichen wissenschaftlichen Resonanzquoten - sprich Zitationen - ein,

\footnotetext{
${ }^{13}$ Es bräuchte allerdings empirische Studien, um die tatsächliche Nachfrage zu klären. Trotz technischer Infrastrukturen wird die Kommentarfunktion innerhalb des wissenschaftlichen Publikationssystems noch nicht breit genutzt, wie es scheint.
}

sondern Daten, die vielmehr den Gebrauchs- und Aufmerksamkeitswert innerhalb und außerhalb der Wissenschaft anzeigen wie z.B. Tweets oder Facebook-Likes. Je höher der Zahlenwert ausfällt, desto relevanter scheint die Publikation, so zumindest der theoretische Aussagewert eines solchen Scores, der von den wissenschaftlichen Großverlagen wie Springer (Bookmetrix), Elsevier oder der Nature Publishing Group bereits in ihr Publikationsangebot integriert wurde. Das jährliche Ranking von Publikationen bei Altmetric ${ }^{14}$ erlaubt einen Einblick in die qualitative Dimension des Scores. Offenkundig handelt es sich bei dieser Maßzahl nicht um einen Indikator für wissenschaftliche Relevanz, aber ebenso wenig um einen Indikator, der den Impact von Forschung auf Gesellschaft abbildet. Stattdessen suggeriert die „Top-100“-Liste von Publikationen mit den höchsten Altmetrics-Werten, dass Themen breit anschlussfähig sind, etwa weil sie umstritten, unterhaltsam oder wissenschaftlich bedeutsam sind (vgl. Franzen 2015b, i.E.). Die vordersten Plätze im Altmetric-Ranking nehmen Publikationen von kuriosen Ergebnissen wie die sonderbare sexuelle Entwicklung eines brasilianischen Höhleninsekts (Platz 14) oder die lebensweltliche Frage nach dem Schokoladenkonsum des Personals auf Krankenstationen (Platz 6) ein. Auch Fälle redaktioneller Unachtsamkeit wie die der fehlenden Löschung eines despektierlichen Autorenkommentars in der Veröffentlichungsversion: "[should we cite the grappy Gabor paper here?] " (Platz 2!) erfreuen sich in den Social Media wie Twitter und Facebook einer besonderen Beliebtheit und steigern dementsprechend den Almetric-Score eines Artikels ungeachtet des wissenschaftlichen Inhalts.

Offensichtlich muss es sich bei dieser Form öffentlicher Bewertung nicht um Urteile zertifizierter Experten handeln. Der Unterschied zu den oben beschriebenen Formen einer indirekten Inklusion in Wissenschaft durch Bildungsangebote oder Medienkonsum besteht darin, dass die Rückäußerungen eines wissenschaftsexternen Publikums - seien es Blogger, PR-Fachleute, Journalisten oder interessierte Laien, die Beiträge bewerten oder rezipieren, indem sie sie u.a. retweeten, liken oder downloaden - für Wissenschaftler unmittelbar relevant werden können. In ihrer aggregierten und nicht-aggregierten Form lassen sich Publikumsresonanzen seitens der Mitglieder der Wissenschaft beobachten und gewinnen auf diese Weise einen möglichen Einfluss

\footnotetext{
14 Siehe das Altmetric-Ranking von 2014 auf: http://www. altmetric.com/top100/2014/ (letzter Zugriff am 19. Mai 2015).
} 
auf die wissenschaftliche Wissensproduktion, zunächst auf die Wissensdarstellung und die Form der Verbreitung. Ein augenfälliges Beispiel für Art und Ton der Publikumsäußerungen geben die Debatten im SozBlog der Deutschen Gesellschaft für Soziologie ab. ${ }^{15}$ Während in der Ära des Druckmediums die Kontrollfunktion über die Veröffentlichung von Beiträgen der Wissenschaft obliegt und gegen Invasoren verteidigt wird (Franzen 2011b), bedeutet die fortschreitende Pluralisierung wissenschaftlicher Publikationsformen eine soziale Öffnung gegenüber Angebot und Nachfrage. Das Bild der relevanten Öffentlichkeit der Wissenschaft ist damit ins Wanken geraten.

Jede öffentliche Kommunikation fungiert als eine Art Spiegel, mit dem soziale Systeme sich selbst beobachten (vgl. Baecker 1996). Die wissenschaftliche Selbstbeobachtung orientiert sich dabei weniger an den Massenmedien, als primär an wissenschaftlichen Zitationen, die in elektronischen Zitationsdatenbanken erfasst sind (Web of Science, Scopus) und individuell wie institutionell reflektiert werden können. Mit dem erweiterten Publikumsradius wissenschaftlicher Kommunikation ändert sich aber nicht nur die Form der Publikation, sondern auch das Gegenüber. Während die Rolle der Leistungsbewertung bislang von Kollegen bzw. Gutachtern besetzt war, lässt sich hier eine tendenzielle Aufweichung erkennen: Spätestens mit der Implementation von Altmetrics verliert sich die Zurechnungsadresse Wissenschaft vs. Nicht-Wissenschaft. Anders als die journalistische Beobachtung von Wissenschaft greifen die neuen technisch-sozialen Infrastrukturen auf die wissenschaftliche Selbstbeobachtung durch. Mittels numerischer Indikatoren lässt sich die Resonanz pro Publikation analog zur Zitationsquote abbilden (Zahl der Downloads, Views, Likes) und für Vergleichszwecke auf unterschiedlichen Aggregationsebenen nutzbar machen. ${ }^{16}$ Der Status der Quelle spielt dabei eine untergeordnete Rolle. Die Präferenzen eines wissenschaftsexternen Publikums könnten somit analog zur Rolle des Kritikers für die wissenschaftliche Leistungserbringung selbst konstitutiv werden. Befördert wird dies durch die forschungspolitische Entwicklung, dem „societal impact“ eine besondere Relevanz in der wissenschaftlichen Leistungsbewertung beizumessen (Bornemann 2012).

\footnotetext{
15 Vgl. z.B. die Diskussion um die Blogpostings von Thomas Kron auf: http://soziologie.de/blog/2015/03/.

${ }^{16} \mathrm{Vgl}$. generell zum kommunikativen Erfolg des quantitativen Vergleichs Heintz (2010).
}

\subsection{Neue Wissensproduzenten}

Während die zuvor skizzierten Entwicklungen auf die Herausbildung eines konstitutiven wissenschaftlichen Publikums abzielen, kristallisiert sich gegenwärtig auch eine Wiederbelebung des nicht-professionellen gesellschaftlichen Wissensproduzenten heraus. Das Schlagwort dafür ist „Citizen Science“. Gemeint ist damit eine Inklusion von Nicht-Wissenschaftlern in die Wissensproduktion, insbesondere in Prozesse der Datengenerierung und Datenauswertung. ${ }^{17}$ Als technologischer Treiber aktueller Citizen Science gelten das Internet im Allgemeinen und die Verbreitung von mobile devices im Besonderen (Roy et al. 2012; Suomela \& Johns 2012; Haklay 2013).

Auch und gerade wissenschaftspolitisch wird die neue Bürgerwissenschaft forciert. Bei der Gründung der European Citizen Science Association (ECSA) formulierte der EU-Forschungskommissar Janez Potocnik 2013 das ehrgeizige Ziel, innerhalb der nächsten fünf Jahre über fünf Millionen Bürger als Citizen Scientists zu motivieren (Helmholtz Zentrum für Umweltforschung 2014). Im Rahmen der Digitalen Agenda der EU-Kommission wird die digital vermittelte Teilnahme von Bürgern an Forschungsprozessen als wichtiger Baustein betrachtet (DG Connect 2013). Mit der jüngst gegründeten digitalen Plattform buergerschaffenwissen.de, die vom BMBF im Rahmen eines großformatigen Verbundprojekts zu Citizen Science gefördert wird, soll Bürgerwissenschaft nun auch in Deutschland digital verankert werden. Auf politischer Ebene wird dies als Reaktion auf einen Partizipationswillen der Bürger gedeutet: Etwa ein Drittel der Deutschen könnte sich einer Umfrage zufolge vorstellen, an einem Citizen Science Projekt mitzuwirken (Wissenschaftsbarometer 2014). Was aber impliziert das Engagement für Citizen Science?

${ }^{17} \mathrm{Zu}$ einem älteren Verständnis von „Citizen Science“ vgl. Irwin (1995). Die aktuelle Dynamik im Bereich der Citizen Science hat die Aufmerksamkeit darauf gelenkt, dass gerade im Bereich von Biodiversitäts- und Umweltforschung Formen der Laienpartizipation in der Forschung nie ganz verschwunden sind. Deren Re-Mobilisierung stellt eine der Zielrichtungen heutiger Citizen Science dar (Roy et al. 2012). Als besonders erfolgreich gilt dabei das vom Imperial College in London getragene Netzwerk für Open Air Laboratories (OPAL). Es dient als Dach für Citizen Science-Projekte in Großbritannien, die von Universitäten, Museen und NGOs organisiert werden. Bislang haben sich 750.000 Freiwillige an OPAL-Projekten beteiligt (Conrad \& Hilchey 2011). 
Mit Citizen Science werden heute mehrheitlich Methoden des wissenschaftsinduzierten Crowdsourcing bezeichnet. Mit Crowdsourcing wird eine Form der Arbeitsteilung beschrieben, in der ein Akteur (typischerweise eine Organisation) eine Aufgabe mithilfe digitaler Medien an eine anonyme Masse delegiert, die diese Aufgabe freiwillig erledigt. Eine Leistung, die normalerweise von Organisationsmitgliedern erbracht werden müsste, wird so ausgelagert (Howe 2010; Estelles-Arolas \& Gonzalez-Ladron-de-Guevara 2012). Crowdsourcing wird durch digitale Infrastrukturen ermöglicht, die eine potenziell globale Adressierung von Interessenten erlauben.

Formen von „Crowd Science“ (Franzoni \& Sauermann 2014) bedeuten somit eine Auslagerung der Datensammlung und -auswertung online an Bürger. Massenhafte Beteiligung und die mehrfache Erledigung einer Aufgabe durch verschiedene Personen sollen die Reliabilität auch dann gewährleisten, wenn die Qualifikation der Teilnehmer an solchen Projekten nicht geprüft werden kann (Roy et al. 2012: 61). „Crowd Science“ ermöglicht datenintensive Großprojekte, die von Laboren mit begrenzter Mitarbeiterzahl kaum umsetzbar wären (Franzoni \& Sauermann 2014: 17). In ihrem Zentrum steht eine wissenschaftliche Projektgruppe, die im Rahmen wissenschaftlicher Organisationen arbeitet und in der zertifizierte Experten Probleme definieren und den Raum möglicher Problemlösungen abstecken. In dieser Form der Citizen Science geht es daher in der Regel darum, den standardisierbaren und oft routineförmigen Teil der Forschungsarbeit an nicht-zertifizierte Akteure zu delegieren.

Einfache Formen der „Crowd Science“ bestehen darin, dass sich Bürger online freiwillig an der Auswertung vorhandenen Datenmaterials beteiligen. Eines der bekanntesten Projekte, das nach diesem Muster funktioniert, ist Galaxy Zoo. Im Rahmen des 2007 gestarteten Projekts besteht die Aufgabe darin, Galaxien auf der Basis von Bildern nach festgelegten Kriterien online zu klassifizieren. Galaxy Zoo ist inzwischen in der Internetplattform Zooniverse aufgegangen (www.zooniverse.org), die von verschieden Universitäten getragen wird und nach eigenen Angaben auf der Beteiligung von 1.343.312 Citizen Scientists basiert (Stand 29.06.2015). Während die Arbeit der Freiwilligen im Zooniverse am heimischen Rechner stattfindet, nutzen andere Projekte Mobilendgeräte - insbesondere in der Biodiversitätsforschung und im Umweltmonitoring. Diese Citizen Science-Projekte nehmen für den Nutzer dabei die Form einer App an. Ein Beispiel dafür ist das Projekt Noise Tube. Die App des Projekts zeichnet den lokalen Geräuschpegel der Umgebung auf, verknüpft diesen mit dem Standort der Nutzer und leitet diese Informationen automatisch an eine Datenbank weiter, um so die Lärmbelastung urbaner Räume zu vermessen.

In besonders anspruchsvollen Wissenschaftsfeldern - etwa der Molekularbiologie - verwenden „Crowd Science“-Projekte Gamification-Ansätze ${ }^{18}$, um Eintrittsschwellen abzusenken. Als Vorreiter gilt dabei das Projekt Foldit (www.foldit.org), bei dem es für die Teilnehmer darum geht, virtuelle Proteine zu „falten“. Was sich für die Spieler als komplexes Puzzle darstellt, das keine spezifischen Vorkenntnisse verlangt, dient den beteiligten Wissenschaftlern als Material, aus dem Erkenntnisse zur Prognose von Proteinstrukturen bzw. dem Design von Proteinen abgeleitet werden können. Im Gegensatz zu Projekten wie Galaxy Zoo, bei denen Problemlösungen klar vorgegeben sind, basiert das Konzept von Foldit darauf, dass Spieler eigene Lösungen für anspruchsvolle Probleme entwickeln können. Damit wird das moderne Deutungsmuster infrage gestellt, dass komplexe wissenschaftliche Aufgaben wie das Proteindesign eine Expertise erfordern, die nur im Kontext wissenschaftlicher Ausbildung erworben werden kann (Haklay 2013: 118f.). In Artikeln, die wissenschaftliche Erfolge auf der Basis der Aktivitäten von Foldit dokumentieren, tauchen Spieler-Gruppen demgemäß auch als kollektive Mitautoren auf (Khatib et al. 2011; Eiben et al. 2012).

Ähnlich wie bei ökonomischen Crowdsourcing-Ansätzen verbindet sich mit "Crowd Science“ die Hoffnung, auf eine nahezu unbegrenzte virtual workforce zurückgreifen zu können, zu der keine formalen Verpflichtungen bestehen, und zugleich denjenigen zeitlichen, räumlichen und finanziellen Begrenzungen beim Zugriff auf Expertise zu entkommen, die Organisationen gesetzt sind (Kleemann et al. 2008; Anderson 2012: 143ff.). Im Unterschied zum wirtschaftlichen Crowdsourcing werden die Crowdworker in der Wissenschaft jedoch für ihre Tätigkeit nicht entlohnt - sie werden nicht als Arbeitskräfte, sondern vielmehr als Citizens adressiert, die durch ihre Tätigkeit einen Beitrag zum Gemeinwohl leisten können und somit der Rolle des „guten Bürgers“ in der Wissensgesellschaft gerecht werden (vgl. Sutter 2005).

Als „Crowd Science“ stellt Citizen Science eine neue Form der „invited participation“ dar. Im Gegensatz zu deliberativen Verfahren wie Bürgerkonferenzen geht es hier nicht um eine Einladung der

18 Darunter versteht man die Integration von Spielmechanismen in nicht-spielerische Kontexte. 
Bürger zum Diskurs, sondern um die Einladung zur Co-Produktion wissenschaftlicher Leistung. Wie aber steht es um Citizen Science als „uninvited participation", die außerhalb von etablierten Institutionen betrieben wird? Deren Quell sind nicht-zertifizierte Formen wissenschaftlicher Praxis, die zwar im Zuge der akademischen Professionalisierung marginalisiert wurden, aber nie völlig verschwunden sind - man denke etwa an Mitglieder in naturkundlichen Vereinigungen oder Stadthistoriker. Der aktuelle Diskurs um Bürgerwissenschaften lenkt die Aufmerksamkeit wieder auf solche residuale Formen von Amateurwissenschaft (Finke 2014).

Die entscheidenden Veränderungsdynamiken durch die Digitalisierung sind hier die folgenden: Digitale Kommunikation erleichtert die Vernetzung von Amateurwissenschaftlern untereinander und stellt eine wechselseitige Sichtbarkeit her, und sie ermöglicht die öffentliche Darstellung von Forschung außerhalb der professionellen Wissenschaft gegenüber einer Öffentlichkeit - etwa durch die Erstellung oder Modifikation von Artikeln auf Wikipedia. Schließlich erweitern sich die Mittel, die Amateurwissenschaftlern zur Verfügung stehen durch Verwendung und Weiterentwicklung open sourcebasierter Soft- und Hardware. ${ }^{19}$ Einige Autoren diagnostizieren daher Möglichkeiten einer neuen Wissenschaft „von unten“, die exkludierte Praktiken der Amateurforschung mit dem Trend zu Open Science und Open Source verknüpft (Delfanti 2010; Haklay 2013; Wylie et al. 2014).

Finke (2014) spricht in diesem Zusammenhang von Citizen Science „proper“, um diese Formen von Amateurwissenschaft von „Crowd Science“ abzugrenzen, die er als Citizen Science „light“ bezeichnet. Während Citizen Science „light“ Inklusion im Kontext bestehender Institutionen befördert, steht Citizen Science „proper“ in der Linie einer gegenkulturellen Praxis, welche bestehende Institutionen infrage stellt (McQuillan 2014). Im Kern kommt in diesen Kategorien erneut die Differenz von „eingeladener Partizipation“ und ,uneingeladener Partizipation" zum Ausdruck (Wynne 2007; Wehling 2012) - diesmal aber mit einer Partizipation von Bürgern, die nicht in Form von Diskursen über Wissenschaft, sondern der (Co-) Produktion von Wissenschaft stattfindet.

\footnotetext{
19 Technisch avancierte Möglichkeiten der Analyse und Manipulation standen der Amateurwissenschaft im 20. Jahrhundert kaum zur Verfügung. Die Konzentration amateurwissenschaftlicher Aktivitäten auf Geisteswissenschaften und klassisch naturkundliche Wissensfelder entsprachen dieser Beschränkung (vgl. Finke 2014).
}

Ein oft angeführtes Paradebeispiel für Citizen Science als „uneingeladener Partizipation“ mit modernen technischen Mitteln stellt die kleine Gemeinschaft der „Biohacker“ (Delfanti 2013) dar, die sich in digitalen Netzwerken wie DIYBio (www.diybio.org) transnational organisiert und koordiniert (Kera 2012; Kelty 2010). Bei Biohackern handelt es sich um Akteure, deren Ziel darin besteht, außerhalb der etablierten institutionellen Strukturen des Wissenschaftssystems lebenswissenschaftliches Wissen zu erwerben, zu teilen und experimentell zu erproben. Die Aktivitäten reichen vom Umweltmonitoring bis hin zu DNA-Sequenzierung. Trotz der vergleichsweise hohen Aufmerksamkeit, die der Biohacking-Szene insbesondere im Kontext der Technikfolgenabschätzung der Synthetischen Biologie zuteilwurde (Bennett et al. 2009), ist die Zahl der „Garagenlabore“, in denen entsprechende Experimente durchgeführt werden, gleichwohl begrenzt. In einer rezenten Analyse der Bewegung kommen Seyfried et al. (2014: 551) dennoch zu dem Schluss, dass im Biohacking ein Prototyp einer demokratischen und offenen Wissenschaft zum Ausdruck kommt.

\subsection{Typologie der Inklusionsmuster}

Die zuvor skizzierten Phänomene indizieren neue Möglichkeiten wissenschaftsspezifischer Partizipation im Kontext digitaler Medien, die von Teilen der STS-community und wissenschaftspolitischen Akteuren - von der EU-Kommission bis hin zu zivilgesellschaftlichen Aktivisten - als Demokratisierung der Wissenschaft gedeutet werden. Diese normativen Interpretationen schließen an übergreifende Diskurse an, die dem Internet im Allgemeinen und dem Web 2.0 im Besonderen ein demokratisierendes Potenzial zusprechen (Schrape 2010). Durch die Anwendung des Demokratisierungsbegriffs, der in seinem semantischen Kern dem politischen System zuzurechnen ist, werden auch andere Gesellschaftsbereiche als Sphären der Macht betrachtet. Die Differenz zwischen Leistungs- und Publikumsrollen wird als Machtasymmetrie interpretiert, wobei Demokratisierung dann eine Relativierung oder gar Einebnung dieser Asymmetrie impliziert (vgl. auch Bora 2005). Gerade im Fall der Wissenschaft ließe sich aber durchaus argumentieren, dass eine exklusive Sphäre zertifizierten Expertenwissens partiell (Collins 2014) funktional notwendig ist (Merton 1942; Weingart 2001). Inwiefern eine Demokratisierung der Wissenschaft dann als normativ wünschenswertes Ziel betrachtet werden kann, ist 


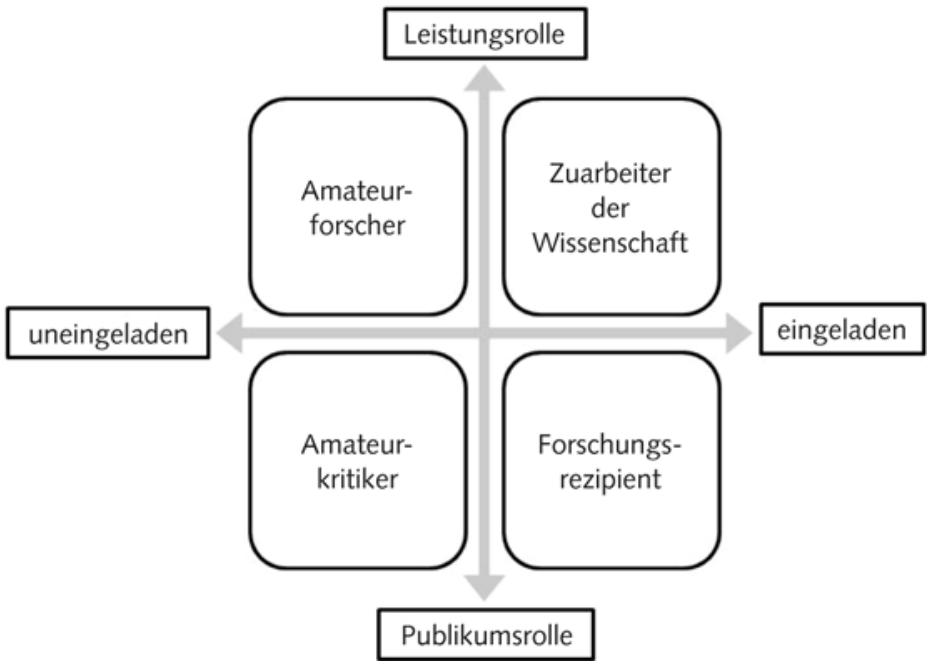

Abb. 1 Typologie der neuen Inklusionsmuster

somit zumindest ambivalent (Nentwich \& König 2012: 188ff.).

In diesem Beitrag schlagen wir gegenüber der normativ aufgeladen Demokratisierungsthese eine gesellschaftheoretische Lesart vor, welche die skizzierten Phänomene der Laienpartizipation an Wissenschaft als neuartige Inklusionsmuster interpretiert. Diese Inklusionsmuster lassen sich von der Warte der wissenschaftlichen Profession wie bereits eingeführt als eingeladene oder uneingeladene Partizipation an der Wissensproduktion fassen. Im ersten Fall wird die Inklusion von Nicht-Wissenschaftlern seitens der Wissenschaft und der Wissenschaftspolitik dezidiert vorangetrieben (eingeladene Partizipation), im zweiten Fall geht der Impuls von den Akteuren selbst aus, die gesellschaftliche Wissensproduktion mitzugestalten (uneingeladene Partizipation).

Mit dem Medienwandel hat sich der Möglichkeitsraum für Akteure außerhalb der akademischen Wissenschaft erheblich erweitert, eigene Wissensbeiträge zu produzieren oder fremde Forschungsbeiträge zu bewerten. Mithilfe der Differenz von eingeladener und uneingeladener Partizipation lassen sich die zuvor beispielhaft vorgestellten Inklusionsmuster der Leistungs- und Publikumsrolle genauer fassen und aufeinander beziehen. Nichtprofessionelle Rollen im Kontext der wissenschaftlichen Leistungsproduktion lassen sich so idealtypisch als Amateurforscher (1) und Zuarbeiter der Wissenschaft (2) bezeichnen. Im Kontext der Leistungsrezeption lässt sich die Rolle des Amateurkritikers (3) von der des Forschungsrezipienten (4) unterscheiden (vgl. Abb. 1).
Als Blaupause der hier entwickelten Typologie der Laieninklusion fungiert das Rollenset des professionellen Wissenschaftlers, der je nach Status und Situation die Rolle des Forschers bzw. Zuarbeiters, des Kritikers und Rezipienten in Personalunion einnimmt. Unsere These auf der Basis der referierten Beobachtungen lautet, dass im gesamten Rollenset der gesellschaftlichen Wissensproduktion soziale Öffnungstendenzen sichtbar werden, die den Laien nicht übergreifend, sondern höchst selektiv in die Wissenschaft inkludieren. In den digital beförderten nicht-professionellen Rollen wird die Rolle des Wissenschaftlers auseinanderdividiert und funktional sub-differenziert (vgl. Abb.1).

Während das Verhältnis zwischen der professionellen Rolle und den beiden Rollenprofilen im Modus eingeladener Partizipation als asymmetrische Kooperationsbeziehung von Wissenschaftlern und Nicht-Wissenschaftlern zu interpretieren ist, lässt sich die Beziehung von Nicht-Wissenschaftlern zu Wissenschaftlern im Modus uneingeladener Partizipation als strukturelle Konkurrenzbeziehung deuten, da sie eine Symmetrisierung von professionellen und nicht-professionellen Rollen impliziert bzw. normativ einfordert (vgl. auch Volkmann 2010: 216f.). Sowohl die Figur des Amateurforschers als auch die des Amateurkritikers repräsentieren formal ein Gegenmodell zur professionellen Praxis, entweder im Hinblick auf die Ebenen der Herstellung oder der Bewertung von Wissen, auf denen seitens der Amateure jeweils andere Relevanzkriterien in Anschlag gebracht werden können.

(1) Die traditionelle Figur des Amateurforschers erlebt eine Renaissance, seit im Kontext von Open 
Science digitale Daten(-sammlungen) veröffentlicht werden, auf die jedermann Zugriff hat. Im Gegensatz zu klassischen Amateurfiguren wie dem Gentleman Scientist stehen Amateurforschern heute auf der Basis digitaler Infrastrukturen einfache Möglichkeiten kollektiver Vernetzung zur Verfügung. Sie stehen nicht mehr vor denjenigen medientechnologischen Hürden, die früheren Formen kollaborativer Amateurforschung kommunikative Grenzen gesetzt hatten (vgl. Mahr 2014). Die strukturell angelegte Konkurrenzbeziehung dieser Rolle zur akademischen Wissenschaft wird z.B. manifest, wenn Amateurforscher ihre Daten und/oder Erkenntnisse den wissenschaftlichen Fachgemeinschaften vorenthalten (Jung 2010) oder umgekehrt kommerziell erfolgreiche Werke von Amateuren seitens der akademischen Zunft verrissen werden. ${ }^{20}$ Die Konkurrenzbeziehung wird stabilisiert, wenn Amateurforscher sich vernetzen und als epistemisches Korrektiv zur akademischen Forschung auftreten (Finke 2015). Die bereits erwähnte Biohacker-Community ist ein prägnantes Beispiel für die explizite Positionierung von Amateurwissenschaft als alternativer wissenschaftlicher Praxisform, welche die Merton'schen Normen unter Rückgriff auf das Hackerethos wiederzubeleben und die Biowissenschaft aus institutionellen Zwängen und externer Einflussnahme zu befreien versucht (Delfanti 2013).

(2) Wenn im wissenschaftspolitischen Diskurs von Citizen Scientists die Rede ist, sind typischerweise nicht Amateurforscher im klassischen Sinne gemeint, sondern Personen, die in Projekte von "Crowd Science“ eingebunden werden. Die hiermit assoziierte Rolle wollen wir deshalb als Zuarbeiter der Wissenschaft bezeichnen. Nicht-Wissenschaftler sind als Bürger aufgerufen, sich an der digitalen Sammlung oder Auswertung von Daten zu beteiligen, während die Formulierung der Fragestellung und die Dateninterpretation weiter in den Händen der projektleitenden Wissenschaftler liegen (Franzoni \& Sauermann 2014). Im Unterschied zur Amateurwissenschaft handelt es sich also der Form nach um eingeladene Partizipation, die professionelle Forschung komplementieren soll. Von Finke (2014) werden jene Formen als Citizen Science light bezeichnet. Zuarbeiter der Wissenschaft werden als Arbeitskräfte gebraucht, um der Datenexplosion in

\footnotetext{
${ }^{20}$ Die Abqualifizierung als ,Amateur“ ist bekanntermaßen nicht allein an den fehlenden professionellen Status geknüpft, sondern das Resultat von „Boundary Work“ (Gieryn 1983), um Wissenschaft von Nicht-Wissenschaft zu trennen.
}

bestimmten Wissenschaftsbereichen gerecht zu werden. Dies betrifft insbesondere diejenigen Fächer, die wie bspw. die Astronomie auf automatisierte Beobachtungsdaten in der Wissensproduktion zurückgreifen. Scientific Literacy ist für diese Hilfstätigkeiten häufig nicht nötig. Das Fehlerrisiko der Datenauswertungen sinkt mit der steigenden Zahl an Teilnehmern in den jeweiligen Projekten. Statt auf individuelle Fähigkeiten wird auf die „Weisheit der Vielen" gesetzt.

(3) Der Amateurkritiker stellt eine ebenfalls für die moderne Wissenschaft ungewohnte und strukturell konkurrierende Rolle dar. Mit der Etablierung entsprechender Online-Plattformen haben ex-post Bewertungen allgemein an öffentlicher Verbreitung gewonnen. Das größte Arsenal an Buchkritiken unterhält heute das Unternehmen Amazon. Alle über Amazon vertriebenen Produkte, von der Waschmaschine bis hin zum wissenschaftlichen Handbuch, werden über das Format der Kundenrezension zugänglich für die öffentliche Kritik durch jedermann. Ungeachtet der inhaltlichen Substanz der vorgebrachten, größtenteils anonymen Bewertung, gewinnen allein die Daten des numerischen Ratings (Fünf-Sterne Skala) Einfluss auf die Sichtbarkeit von Produkten etwa auf Fachbücher. Über den technisch eingebauten Empfehlungsdienst wird das Rezeptionsverhalten von Konsumenten, aber eben auch von Wissenschaftlern bis zu einem gewissen Grad gesteuert, da er die Sichtbarkeit von Publikationen beeinflusst. Neben jenen Formen kumulierter Bewertung über die Aggregation von Einzelurteilen im Buchbereich, sind es im wissenschaftlichen Zeitschriftenwesen elektronische Kommentarfunktionen, die theoretisch von Nicht-Wissenschaftlern genauso wie von Wissenschaftlern für eine kritische Betrachtung der wissenschaftlichen Inhalte genutzt werden könnten. ${ }^{21} \mathrm{Zu}$ erwähnen sind darüber hinaus kollaborative Unternehmungen wie die Plagiatsdokumentation in Wikis, z.B. Guttenplag, oder Blogs, z.B. Retraction Watch, die quasi eine Monitoring-Funktion für wissenschaftliche Integrität einnehmen und somit den Nicht-Wissenschaftler potenziell sogar in die Bewertung wissenschaftlicher (Fehl-)Leistungen integrieren.

(4) Die vierte und letzte Rolle, die wir mit den Forschungsrezipienten benennen möchten, ist nicht identisch mit der wissenschaftsinteressierten Öf-

\footnotetext{
21 Sozio-technische Infrastrukturen der Bewertung befördern zudem, wie das Beispiel von TripAdvisor im Touristikbereich zeigt (Jeacle \& Carter 2011), den Autoritätsverlust des einzelnen Experten zugunsten der (authentischen) Sicht der Nutzer.
} 
fentlichkeit als Kollektiv, deren Teilnehmer über Wissenschaftskommunikationsaktivitäten oder Bildungsangebote in die Wissenschaft (indirekt) inkludiert werden. Stattdessen handelt es sich beim Forschungsrezipienten analog zum vorherigen Typus um eine aktive Publikumsrolle. Über das Teilen, Liken, Downloaden, Re-Tweeten oder Sichten von wissenschaftlichen Produkten im Web 2.0 entstehen nutzergenerierte Daten, die ungeachtet des Status des Rezipienten und seiner Intentionen in die Nutzungsstatistiken wissenschaftlicher Verlage mit einfließen. Wenn mit der zunehmenden Implementation von Altmetrics im wissenschaftlichen Publikationssystem der so erfasste Gebrauchswert von Publikationen zum qualitativen Vergleichsmaßstab innerhalb der Wissenschaft avanciert, werden die Grenzen zwischen Wissenschaft und Nicht-Wissenschaft tatsächlich durchlässig (Franzen 2015b, i.E.). Der anonyme Forschungsrezipient ist für den Wissenschaftler eine willkommene Adresse, wenn es heute verstärkt darum geht, eine Reichweitenmaximierung der eigenen Forschungsaktivität zu erreichen. Das Erzielen von Aufmerksamkeit bedeutet auch in der Wissenschaft die erwünschte Anerkennung (Schroer 2014), und die Zirkulation von Themen über verschiedene Kanäle scheint als ein probates Mittel. In diesem Sinne ordnen wir den Forschungsrezipienten analog zum Zuarbeiter der Wissenschaft der Dimension der eingeladenen und komplementären Partizipation zu, während die beiden Typen Amateurforscher und Amateurkritiker den gegenkulturellen Pol der uneingeladenen Partizipation repräsentieren.

An dieser Stelle soll nochmals hervorgehoben werden, dass es sich bei den skizzierten Typen um Idealtypen handelt, „gewonnen durch einseitige Steigerung eines oder einiger Gesichtspunkte und durch Zusammenschluß einer Fülle von diffus und diskret, hier mehr, dort weniger, stellenweise gar nicht, vorhandenen Einzelerscheinungen, [...] zu einem in sich einheitlichen Gedankenbilde. In seiner begrifflichen Reinheit ist dieses Gedankenbild nirgend in der Wirklichkeit empirisch vorfindbar", es leistet aber „[f]ür den Zweck der Erforschung und Veranschaulichung [...] vorsichtig angewendet seine spezifischen Dienste" (Weber 1991: 73f.; Hervorh. i.O.). Der heuristische Zweck der von uns herausgearbeiteten Typen besteht in der Sensibilisierung für Veränderungsdynamiken, die sich für die Wissenschaft im Zuge der Digitalisierung abzeichnen.

\section{Fazit und Ausblick: Auf dem Weg in die nächste Gesellschaft?}

Mit der sachlichen Ausdifferenzierung der modernen Wissenschaft im 19. Jahrhundert ging ihre soziale Abriegelung durch Fachsprachen und die Herausbildung eines selbstreferentiellen Kommunikationszusammenhangs einher. Die Wissenschaft wurde zu einem System, das sowohl ohne genuine Publikumsrolle auskam als auch kaum mehr einen Ort für sekundäre Leistungsrollen bot. Erst im Zuge einer "Medialisierung der Wissenschaft" (Weingart 2012) begann sich diese soziale Exklusivität langsam zu relativieren. Es zeigt sich, dass die rezente Entwicklung digitaler Medien hin zum Web 2.0 nun zur Entwicklung prototypischer Formen führt, die diese Entwicklung radikalisieren: Wir beobachten Ansätze zur Ausdifferenzierung nichtprofessioneller Rollen in der Wissenschaft, die sich hinsichtlich des jeweils wirksamen Inklusionsmechanismus von eingeladener und uneingeladener Partizipation zu vier verschiedenen Inklusionsprofilen analytisch verdichten lassen: 1) Amateurforscher, 2) Zuarbeiter der Wissenschaft, 3) Amateurkritiker und 4) Forschungsrezipient.

Bei diesen vier Inklusionsprofilen handelt es sich um Idealtypen, zwischen denen sich die neuen Formen der Inklusion empirisch aufspannen. Die Annahme lautet, dass die vorgestellten Inklusionsprofile für Nicht-Wissenschaftler bislang nicht die Wissenschaft als Ganze betreffen. Vielmehr haben wir es mit prototypischen Erscheinungsformen sozialer Öffnung zu tun, die auf fachgebietsspezifischen Herstellungs- und Darstellungsbedingungen von Wissen aufbauen. Das Erreichen eines außerwissenschaftlichen Publikums ist thematisch gebunden und eng an eine Informalisierung wissenschaftlicher Kommunikation geknüpft. Laien-Rezensionen sind bei Publikationstypen zu erwarten, die allgemeinverständlich gehalten sind und auf alltagsweltliches Interesse stoßen. „Crowd Science“ ist eher bei solchen wissenschaftlichen Feldern nachgefragt, in denen massenhafte Datensammlung und/ oder -auswertung sinnvoll erscheinen. Neue Formen einer Amateurwissenschaft „von unten“ sind bei esoterischen und labororientierten Wissensgebieten schwer vorstellbar - oder erfordern, wie der Fall der Biohacker zeigt, eine Absenkung technischer Zugangshürden.

Gegenwärtig können unsere Beobachtungen zu den neuen Inklusionsmustern nur den Status qualitativer Strukturgeneralisierungen beanspruchen. Ihre gesellschaftstheoretische Relevanz entfalten sie erst vor der Annahme, dass der digitale Wandel keine 
flüchtige Modeerscheinung ist, sondern ein radikaler Transformationsprozess. Die abgeleiteten Inklusionsmuster beruhen auf digitalen Netzwerkstrukturen, die Informalisierungstendenzen befördern. Sie demonstrieren, dass Inklusion in die wissenschaftliche Wissensproduktion nicht mehr exklusiv an formale Qualifikationen oder das Verständnis hochspezialisierter Fachsprachen gebunden sein muss. Auf der Basis digitaler Infrastrukturen können auch nicht-zertifizierte Akteure an der Wissensproduktion teilnehmen. Diese Teilnahme betrifft zunächst die Darstellungsebene: die Rezeption, Verbreitung, das numerische Rating und eventuell die inhaltliche Kommentierung wissenschaftlicher $\mathrm{Pu}$ blikationen. Je stärker sich die wissenschaftliche Kommunikation in Richtung „Open Access“ bewegt, Publikationsformen sich pluralisieren und Altmetrics an Bedeutung in der wissenschaftlichen Leistungsbewertung gewinnt, desto eher ist zu erwarten, dass wissenschaftliche Veröffentlichungen sich an ein breiteres Publikum wenden und von diesem auch rezipiert werden.

Analog dazu sind Öffnungsprozesse bei der Herstellung von Wissen zu registrieren: Im Kontext von "Crowd Science“ erfordert Partizipation an der Wissensproduktion weder eine formale Qualifikation noch setzt sie ein Interesse an wissenschaftlichen Fragestellungen oder ein Bedürfnis nach demokratisierter Expertise zwingend voraus. Inwiefern Digitalisierung neue Möglichkeiten für Forschungen außerhalb bestehender Institutionen befördert, zeigen exemplarisch die Biohacker. Solche Formen der Amateurforschung können als durchaus konsequente Realisierung der Leitidee einer Open Science verstanden werden, die die sozialen Schließungsmechanismen der modernen Wissenschaft überwinden möchte (Delfanti 2013).

Die soziale Öffnung der Wissenschaft, wie sie politisch-administrativ durch Open Science und Science 2.0 befördert und theoriepolitisch auch von Collins und Evans (2002) als Third Wave der Science Studies problematisiert wird, ist theoretisch brisant. Für die Differenzierungstheorie ergibt sich die Provokation, dass die dargelegte Erweiterung von Rollenprofilen eher dem Wissenschaftsbild rezenter auf Transdisziplinarität abstellender - Zeitdiagnosen (von „postnormal science“ bis „Mode 2“) entspricht als dem differenzierungstheoretischen Bild ${ }^{22}$ selbstreferentieller Kommunikationskreisläufe und einer über Organisationen und Professionen abge-

22 Ein solches Bild der Wissenschaft wurde in der Second Wave der Science Studies (Collins \& Evans 2002) als idealisierte Selbstbeschreibung zurückgewiesen. sicherten funktionalen Autonomie. Unter den Bedingungen funktionaler Differenzierung erscheint eine restriktive Inklusionsordnung der Wissenschaft entsprechend als unveränderlicher Tatbestand.

$\mathrm{Zu}$ prüfen ist allerdings, inwiefern sich diese beiden Perspektiven mit Blick auf unseren Gegenstand integrieren lassen. Einen zentralen Baustein für diese Integration liefert die von Luhmann (1997) vorbereite und von Baecker (2007) ausbuchstabierte Theorie des medialen Epochenwandels. Die Grundidee ist, dass bestehende Gesellschaftsformationen insbesondere durch disruptive Medieninnovationen infrage gestellt werden: „Jedes in der Evolution der Gesellschaft neu auftretende Verbreitungsmedium der Gesellschaft attrahiert neue Möglichkeiten der Kommunikation, das heißt des Erreichens und Verstehens neuer Kreise von Adressaten, und bedroht damit die bisherige Struktur und Kultur, die bisherigen Institutionen, Konventionen und Routinen, die auf die Modalitäten der älteren Verbreitungsmedien eingestellt sind." (Baecker 2015: 4, Hervorhebung SD \& MF)

Wenn man Verbreitungsmedien nicht nur in ihrer destabilisierenden Wirkung in den Blick nimmt, sondern auch und gerade als evolutionäre Errungenschaften auffasst, denen das Potenzial innewohnt, neue Formen gesellschaftlicher Strukturbildung zu ermöglichen (Luhmann 1997: 515f.; vgl. auch Fuhse 2010), so geben die hier referierten Beobachtungen Anlass, über gesellschaftliche Strukturtransformationen angesichts der digitalen Revolution nachzudenken. „Die Einführung der Sprache konstituierte die Stammesgesellschaft, die Einführung der Schrift die antike Hochkultur, die Einführung des Buchdrucks die moderne Gesellschaft und die Einführung des Computers die nächste Gesellschaft", so Dirk Baecker (2007: 7). Die als medial bedingter Epochenbruch postulierte und skizzierte „nächste Gesellschaft“ zeichnet sich dadurch aus, dass die funktionale Differenzierungsstruktur der Moderne durch technisch vermittelte Netzwerkstrukturen überlagert wird, welche zunehmend Integrations- und Koordinationsfunktionen übernehmen (vgl. auch Castells 2004). Die Figur der Überlagerung (nicht: Ablösung) ist hier höchst relevant: Die neuen, durch digitale „Infrastrukturen des Kollektiven “ (Stäheli 2012) vermittelten sozialen Muster substituieren bisherige Ordnungen nicht, sondern stellen selektive funktionale Äquivalente ,in zunächst möglicherweise marginalen, dann zunehmend zentralen Bereichen“ (Baecker 2015: 6) bereit.

Die provokante These der „nächsten Gesellschaft“ liefert eine Hintergrundfolie, um unsere prototypi- 
schen Fälle neuer Inklusionsmuster tatsächlich als Prototypen zu interpretieren, als realexperimentelle Vorreiter einer Wissenschaft der nächsten Gesellschaft. Sie demonstrieren, wie eine Wissenschaft aussehen könnte, die in den Sog digitaler Netzwerke gerät, welche es ermöglichen, funktionssystemische Leistungserbringung über Organisationsund Professionsgrenzen hinaus herzustellen und so eine soziale Entkopplung von Kommunikation zu befördern. So erscheint eine dezentrierte Wissensproduktion vorstellbar, die nicht mehr exklusiv durch die Berufsrolle des Wissenschaftlers strukturiert ist, der verschiedene wissenschaftliche Funktionen (Forscher, Zuarbeiter, Kritiker, Rezipient) in sich bündelt und qua professioneller Autorität monopolisiert. Das Argument ist dabei nicht, dass Experten durch Laien substituiert werden, sondern dass in allen vier Typen die Unterscheidung zertifizierte/nicht-zertifizierte Expertise (vgl. Collins \& Evans 2002) insgesamt (unterschiedlich weit) in den Hintergrund rückt.

Die These eines digital basierten gesellschaftsstrukturellen Wandels ist in ihrer Gänze empirisch nicht überprüfbar. ${ }^{23}$ Sie ist aber sehr wohl als heuristisches Instrument zu gebrauchen. Ihr wesentlicher Schwachpunkt ist ihre bislang (sowohl bei Luhmann als auch bei Baecker) ausstehende Spezifizierung dessen, was sich hinter den suggerierten Umbrüchen genau verbirgt und welche Strukturen als funktionale Äquivalente der durch Medienwandel destabilisierten Strukturen gelten könnten. Insofern bietet unsere Analyse hier eine mehrfache Konkretisierung an: Am Fall eines Funktionssystems (der Wissenschaft) beziehen wir uns auf eine spezifische Struktur, die digital destabilisiert werden könnte (exklusive Bindung der Leistungserbringung durch professionalisierte Rollenträger). Mit der Herausarbeitung von vier Inklusionsmustern zeigen wir ein Spektrum möglicher funktionaler Äquivalente auf, die diese Struktur überlagern. Überlagerung heißt demnach, dass die professionelle Rolle des Wissenschaftlers auseinanderdividiert und funktio-

\footnotetext{
${ }^{23}$ Die These einer „nächsten Gesellschaft" erscheint weit weniger unwahrscheinlich und spekulativ als man meinen könnte, wenn man die denkbaren Alternativen in Betracht zieht, die sich aus der systemtheoretischen Differenzierungstheorie ableiten lassen. Entweder müsste man annehmen, dass der aktuelle digitale Wandel weniger durchgreifend ist als alle anderen Medienrevolutionen zuvor (Sprache, Schrift, Buchdruck) oder aber - wie Anna Henkel (2010) richtigerweise ausführt - von einem Zusammenbruch funktionaler Differenzierung ausgehen, obne dass sich neue sozialstrukturelle Muster als funktionale Äquivalente herausbilden.
}

nal sub-differenziert wird. Mit den auf digitalen Infrastrukturen aufbauenden Inklusionsmustern werden jeweils Kooperations- oder Konkurrenzbeziehungen zur professionellen Wissenschaft geknüpft, welche eine stabilisierende oder destabilisierende Wirkung entfalten können.

Vor dem Hintergrund der sich abzeichnenden Entwicklung scheint es geboten, das Transformationspotenzial des Medienwandels zumindest als ernstzunehmenden Ausgangspunkt soziologischer Theoriebildung und empirischer Forschung zu begreifen.

\section{Literatur}

Abels, G. \& A. Bora, 2004: Demokratische Technikbewertung. Bielefeld: Transcript.

Anderson, C., 2012: Makers. The New Industrial Revolution. New York: Crown Business.

Auer, P. \& H. Baßler (Hrsg.), 2007: Reden und Schreiben in der Wissenschaft. Frankfurt am Main, New York: Campus.

Baecker, D., 1996: Oszillierende Öffentlichkeit. S. 89-107 in: R. Maresch (Hrsg.), Medien und Öffentlichkeit. Positionierungen, Symptome, Simulationsbrüche. München: Boer.

Baecker, D., 2007: Studien zur nächsten Gesellschaft. Frankfurt am Main: Suhrkamp.

Baecker, D., 2015: Ausgangspunkte einer Theorie der Digitalisierung. https:/catjects.files.wordpress.com/2015/ 06/ausgangspunkte_theorie_digitalisierung.pdf (29.6.2015).

Bauer, M. W., 2012: Public Attention to Science, 1820-2010 - a 'Longue Duree' Picture. S. 35-58 in: S. Rödder, M. Franzen, \& P. Weingart (Hrsg.), The Sciences' Media Connection - Public Communication and its Repercussions, Sociology of the Sciences Yearbook 28, Dordrecht u.a.: Springer.

Bauer, M. W., N. Allum \& S. Miller, 2007: What Can We Learn from 25 Years of PUS Survey Research? Liberating and Expanding the Agenda. Public Understanding of Science 16: 79-95.

Ben-David, J., 1991: Scientific Growth: Essays on the Social Organization and Ethos of Science. Berkeley: University of California Press.

Bennett, G., N. Gilman, A. Stavrianakis \& P. Rabinow, 2009: From Synthetic Biology to Biohacking: Are We Prepared? Nature Biotechnology 27: 1109-1111.

Bensaude-Vincent, B. 2001: A Genealogy of the Increasing Gap Between Science and the Public. Public Understanding of Science 10: 99-113.

Bogner, A., 2010: Partizipation als Laborexperiment. Paradoxien der Laiendeliberation in Technikfragen. Zeitschrift für Soziologie 39: 87-105.

Bora, A., 2005: „Partizipation“ als politische Inklusionsformel. S. 15-34 in: C. Gusy (Hrsg.), Inklusion und Partizipation. Politische Kommunikation im historischen Wandel. Frankfurt am Main, New York: Campus. 
Bora, A., 2010: Technoscientific Normativity and the "Iron Cage" of Law. Science, Technology \& Human Values 35: 3-28.

Bornmann, L., 2012: Measuring the Societal Impact of Research: Research is Less and Less Assessed on Scientific Impact Alone - We Should Aim to Quantify the Increasingly Important Contributions of Science to Society. EMBO Report 13: 673-676.

Bucchi, M. \& B. Trench, 2014: Handbook of Public Communication of Science and Technology. 2. Aufl. London, New York: Routledge.

Brown, P., 2007: Toxic Exposures. Contested Illness and the Environmental Health Move-ment. New York: Comlumbia University Press.

Burzan, N., B. Lökenhoff, B., U. Schimank, U. \& N. M. Schöneck, 2008: Das Publikum der Gesellschaft. Inklusionsverhältnisse und Inklusionsprofile in Deutschland. Wiesbaden: VS.

Castells, M., 2004: Das Informationszeitalter: Wirtschaft; Gesellschaft; Kultur. Teil 1.: Der Aufstieg der Netzwerkgesellschaft. Opladen: Leske und Budrich.

Collins, H. \& R. Evans, 2002: The Third Wave of Science Studies: Studies of Expertise and Experience. Social Studies of Science 32: 235-296.

Conrad, C.C. \& K.G. Hilchey, 2011: A Review of Citizen Science and Community-based Environmental Monitoring: Issues and Opportunities. Environmental Monitoring and Assessment 176: 273-291.

Delfanti, A., 2010: Users and Peers. From Citizen Science to P2P Science. Journal of Science Communication 9 E.

Delfanti, A., 2013: Biohackers. The Politics of Open Science. London: Pluto Press.

DG Connect, 2013: Digital Science in Horizon 2020.

Eiben, C.B., J.B. Siegel, J.B. Bale, S. Cooper, F. Khatib, B.W. Shen, Foldit Players, B.L. Stoddard, Z. Popovic \& D. Baker, 2012: Increased Diels-Alderase Activity Through Backbone Remodeling Guided by Foldit Players. Nature Biotechnology 30: 190-192.

Epstein, S., 1995: The Construction of Lay Expertise: AIDS Activism and the Forging of Credibility in the Reform of Clinical Trials. Science, Technology \& Human Values 20: 408-437.

Epstein S., 2011: Measuring Success: Scientific, Institutional, and Cultural Effects of Patient Advocacy. S. 257-277 in: B. Hoffman, N. Tomes, R. Grob \& M. Schlesinger (Hrsg.), Patients as Policy Actors. New Brunswick: Rutgers University Press.

Estelles-Arolas, E. \& F. Gonzalez-Ladron-de-Guevara, 2012: Towards an Integrated Crowdsourcing Definition. Journal of Information Science 38: 189-200.

Finke, P. (Hrsg.), 2015: Freie Bürger, freie Forschung. Die Wissenschaft verlässt den Elfenbeinturm. München: Oekom.

Finke, P., 2014: Citizen Science. Das unterschätzte Wissen der Laien. München: Oekom.

Fox, F., 2012: Practitioner's Perspective: The Role and Function of the Science Media Centre. S. 257-270 in: S. Rödder, M. Franzen \& P. Weingart (Hrsg.), The Sciences' Media Connection - Public Communication and its Repercussions, Sociology of the Sciences Yearbook 28, Dordrecht u.a.: Springer.

Franzen, M., 2011a: Breaking News. Wissenschaftliche Zeitschriften im Kampf um Aufmerksamkeit. Baden Baden: Nomos.

Franzen, M., 2011b: Medien und ihre Resonanzeffekte in der Wissenschaft. WPK-Quarterly 9: 15-19.

Franzen, M. (i. E.), 2015a: Science between Trust and Control: Non-Reproducibility in Scholarly Publishing. S. 468-485 in: H. Atmanspacher \& S. Maasen (Hrsg.), Handbook: Reproducibility: Principles, Problems, Practices. New York: Wiley.

Franzen, M. (i. E.), 2015b: Der Impact Factor war gestern. Altmetrics und die Zukunft der Wissenschaft. Sonderband Soziale Welt

Franzoni, C. \& H. Sauermann, 2014: Crowd science: The organization of Scientific Research in Open Collaborative Projects. Research Policy 43: 1-20.

Fuhse, J., 2010: Welche kulturellen Formationen entstehen in mediatisierten Kommunikationsnetzwerken? S. 31-54 in: J. Fuhse \& C. Stegbauer (Hrsg.), Kultur und mediale Kommunikation in sozialen Netzwerken. Wiesbaden: VS.

Funtowicz, S.0. \& J.R. Ravetz, 1993: Science for the PostNormal Age. Futures 25: 739-755.

Gerhards, J., 2001: Der Aufstand des Publikums. Eine systemtheoretische Interpretation des Kulturwandels in Deutschland zwischen 1960 und 1989. Zeitschrift für Soziologie 30: 163-184.

Gibbons, M., C. Limoges, H. Nowotny, S. Schwartzman, P. Scott \& M. Trow, 1994: The New Production of Knowledge. The Dynamics of Science and Research in Contemporary Societies. London: Sage.

Gieryn, T. F., 1983: Boundary-Work and the Demarcation of Science from Non-Science: Strains and Interests in Professional Ideologies of Scientists. American Sociological Review 48: 781-795.

Gläser, J., 2006: Wissenschaftliche Produktionsgemeinschaften. Die soziale Ordnung der Forschung. Frankfurt am Main, New York: Campus.

Grohs, G., 1964: Wandlungen der Sozialen Rolle des Kunstkritikers. Archives Européennes de Sociologie 5: 311-323.

Gross, L., 2012: Practitioner's Perspective: Science as a Public Resource: Rules of Enga-gement. S. 353-360 in: S. Rödder, M. Franzen \& P. Weingart (Hrsg.), The Sciences' Media Connection - Public Communication and its Repercussions. Sociology of the Sciences Yearbook 28. Dordrecht: Springer.

Guston, D. H., 2006: Between Politics and Science: Assuring the Integrity and Productivity of Research. Cambridge: Cambridge University Press.

Haklay, M., 2013: Citizen Science and Volunteered Geographic Information: Overview and Typology of Participation. S. 105-122 in: D. Sui, S. Elwood \& M. Goodchild (Hrsg.), Crowdsourcing Geographic Knowledge. Dordrecht: Springer.

Heintz, B., 2010: Numerische Differenz. Überlegungen zu einer Soziologie des (quantitativen) Vergleichs. Zeitschrift für Soziologie. 39: 162-181. 
Helmholtz Zentrum für Umweltforschung, 2014: Bürger schaffen Wissen. Wie Citizen Science in Deutschland ausgebaut werden soll. Pressemitteilung vom 07.07.2014. http://www.ufz.de/index.php?de=32981 (03.11.2014).

Henkel, A., 2010: Verbreitungsmedien, Organisation und die nächste Gesellschaft. S. 83-111 in: S. Roth, L. Scheiber \& R. Wetzel (Hrsg.), Organisation multimedial. Zum polyphonen Programm der nächsten Organisation. Heidelberg: Auer.

Hochadel, O., 2003: Elektrizität in der deutschen Aufklärung. Göttingen: Wallstein.

Howe, J., 2010: Crowdsourcing. Why the Power of the Crowd is Driving the Future of Business. http:// crowdsourcing.typepad.com/ (25.4.2013).

Irwin, A., 1995: Citizen Science. A Study of People, Expertise, and Sustainable Development. London, New York: Routledge.

Jasanoff, S., 2004: States of Knowledge: The Co-Production of Science and Social Order. London, New York: Routledge.

Jasanoff, S., 2011: Constitutional Moments in Governing Science and Technology. Science and Engineering Ethics 17: 621-638.

Jeacle, I. \& C. Carter, 2011: In TripAdvisor We Trust: Rankings, Calculative Regimes and Abstract Systems. Accounting, Organizations and Society 36: 293-309.

Jung, M., 2010: „Heimathirsche“. Hobbyarchäologen zwischen Hedonismus und Professionalisierung. Münster: Waxmann.

Junge, T., 2008: Gouvernementalität der Wissensgesellschaft. Politik und Subjektivität unter dem Regime des Wissens. Bielefeld: Transcript.

Kelty, C.M., 2010: Outlaw, Hackers, Victorian Amateurs. Diagnosing Public Participation in the Life Sciences Today. Journal of Science Communication $9 \mathrm{E}$.

Kera, D., 2012: Hackerspaces and DIYbio in Asia. Connecting Science and Community with Open Data, Kits and Protocols. Journal of Peer Production 2 E.

Khatib, F., DiMaio, Foldit Contenders Group, Foldit Void Crushers Group, Frank, S. Cooper, M. Kazmierczyk, M. Gilski, S. Krzywda, H. Zabranska, I. Pichova, J. Thompson, Z. Popovic, M. Jaskolski \& D. Baker, 2011: Crystal Structure of a Monomeric Retroviral Protease Solved by Protein Folding Game Players. Nature Structural \& Molecular Biology 18: $1175-$ 1177.

Kitcher, P., 2011: Science in a Democratic Society. Amherst, NY: Prometheus.

Kleemann, F., G.G. Voß \& K. Rieder, 2008: Crowdsourcing und der arbeitende Konsument. Arbeits- und Industriesoziologische Studien 1: 29-44.

Lemmens Medien GmbH, 2013: citizenscience:germany. Bürger forschen mit. Berlin.

Lin, J. \& M. Fenner, 2013: Altmetrics in Evolution: Defining and Redefining the Ontology of Article-Level Metrics, ISQ 25: 20-26.

Luhmann, N., 1990: Die Wissenschaft der Gesellschaft. Frankfurt am Main: Suhrkamp.

Luhmann, N., 1997: Die Gesellschaft der Gesellschaft. Frankfurt am Main: Suhrkamp.
Luhmann, N., 1999: Funktionen und Folgen formaler Organisation, 5. Aufl. Berlin: Duncker \& Humblot.

Mahr, D., 2014: Citizen Science. Partizipative Wissenschaft im späten 19. und frühen 20. Jahrhundert. Baden-Baden: Nomos.

McQuillan, D., 2014: The Countercultural Potential of Citizen Science. Journal of Media and Culture 17E.

Merton, R. K, 1942: The Normative Structure of Science. S. 267-278 in: N.W. Storer (Hrsg.), The Sociology of Science (1973). Chicago: University of Chicago Press.

Nentwich, M. \& R. König, 2012: Cyberscience 2.0. Research in the Age of Digital Social Networks. Frankfurt am Main, New York: Campus.

Nowotny, H., P. Scott \& M. Gibbons (2001): Re-Thinking Science. Knowledge and the Public in an Age of Uncertainty. Cambridge: Cambridge University Press.

Oevermann, U., 2005: Wissenschaft als Beruf. Die Professionalisierung wissenschaftlichen Handelns und die gegenwärtige Universitätsentwicklung. Die Hochschule 14: 15-51.

Pfadenhauer, M., 2003: Professionalität. Eine wissenssoziologische Rekonstruktion institutionalisierter Kompentenzdarstellungskompetenz. Opladen: Leske und Budrich.

PLoS Medicine Editors, 2006: Editorial: The Impact Factor Game. PLoS Medicine 3, e291.

Priem, J., 2013: Beyond the Paper. Nature 495: 437-440.

Reimer, J. \& M. Ruppert, 2012: GuttenPlag-Wiki und Journalismus. Das Verhältnis eines neuen Medienakteurs im Social Web zu den traditionellen Massenmedien. S. 303-329 in: U. Dolata \& J.-F. Schrape (Hrsg.), Internet, Mobile Devices und die Transformation der Medien. Radikaler Wandel als schrittweise Rekonfiguration. Berlin: Edition Sigma.

Roy, H.E., M. Pocock, C.D. Preston, D.B. Roy, J. Savage, J.C. Tweddle \& L. Robinson, 2012: Understanding Citizen Science and Environmental Monitoring. Final Report on behalf of UK Environmental Observation Framework.

Schimank, U., 2012: Wissenschaft als gesellschaftliches Teilsystem. S. 113-123 in: S. Maasen, M. Kaiser, M. Reinhart \& B. Sutter (Hrsg.), Handbuch Wissenschaftssoziologie. Wiesbaden: Springer VS.

Schrape, J.-F., 2010: Neue Demokratie im Netz? Eine Kritik an den Visionen der Informationsgesellschaft. Bielefeld: Transcript.

Schroer, M., 2014: Soziologie der Aufmerksamkeit. Grundlegende Überlegungen zu einem Theorieprogramm. Kölner Zeitschrift für Soziologie und Sozialpsychologie 66: 193-218.

Seyfried, G., L. Pei \& M. Schmidt, 2014: European Do-ItYourself (DIY) Biology: Beyond the Hope, Hype and Horror. BioEssays: News and Reviews in Molecular, Cellular and Developmental Biology 36: 548-551.

Shapin, S. \& S. Schaffer, 1985: Leviathan and the AirPump. Hobbes, Boyle, and the Experimental Life. Princeton: Princeton University Press.

Shirky, C., 2008: Here Comes Everybody. The Power of Organizing Without Organizations. New York: Penguin Books. 
Stäheli, U., 2012: Infrastrukturen des Kollektiven. Alte Medien, neue Kollektive. Zeitschrift für Medien- und Kulturforschung 3: 99-116.

Stichweh, R., 1988a: Differenzierung des Wissenschaftssystems. S. 45-115 in: R. Mayntz, B. Rosewitz, U. Schimank \& R. Stichweh (Hrsg.), Differenzierung und Verselbständigung Frankfurt am Main, New York: Campus.

Stichweh, R., 1988b: Inklusion in Funktionssysteme der modernen Gesellschaft. S. 261-293 in: R. Mayntz, B. Rosewitz, U. Schimank \& R. Stichweh (Hrsg.), Differenzierung und Verselbständigung. Frankfurt am Main, New York: Campus.

Suomela, T. \& E. Johns, 2012: Citizen Participation in the Biological Sciences. A Literature Review of Citizen Science. Library and Information Science Commons. http://trace.tennessee.edu/ccisymposium/2012/poster/8/ (11.03.2015).

Sutter, B., 2005: Von Laien und guten Bürgern: Partizipation als politische Technologie. S. 220-240 in: A. Bogner \& H. Torgersen (Hrsg.), Wozu Experten? Ambivalenzen der Beziehung von Wissenschaft und Politik. Wiesbaden: VS.

Toffler, A., 1980: The Third Wave. New York: Bantam Books.

Volkmann, U., 2010: Sekundäre Leistungsrolle. Eine differenzierungstheoretische Einordnung des Prosumenten am Beispiel des „Leser-Reporters“. S. 206-220 in: B. Blättel-Mink \& K.-U. Hellmann (Hrsg.), Prosumer revisited. Zur Aktualität einer Debatte. Wiesbaden: VS.

Weber, M., 1991 [1904]: Die „Objektivität“ sozialwissenschaftlicher und sozialpolitischer Erkenntnis. S. 21101 in: M. Sukale (Hrsg.), Max Weber: Schriften zur Wissenschaftslehre. Stuttgart: Reclam.
Wehling, P., 2012: From Invited to Uninvited Participation (and Back?): Rethinking Civil Society Engagement in Technology Assessment and Development. Special Issue on Participatory Technology Assessment of Poiesis $\&$ Praxis. International Journal of Technology Assessment and Ethics of Science 9: 43-60.

Weingart, P., 2001: Die Stunde der Wahrheit? Weilerswist: Velbrück.

Weingart, P., 2005: Die Wissenschaft der Öffentlichkeit. Essays zum Verhältnis von Wissenschaft, Medien und Öffentlichkeit. Weilerswist: Velbrück.

Weingart, P., 2012: The Lure of the Mass Media and its Repercussions on Science. Theoretical Considerations on the 'Medialization of Science'. S. 17-32 in: S. Rödder, M. Franzen \& P. Weingart (Hrsg.), The Sciences' Media Connection - Public Communication and its Repercussions. Sociology of the Sciences Yearbook 28. Dordrecht: Springer.

Whitley, R., 2000: The Intellectual and Social organization of the Sciences. 2. Aufl. Oxford, New York: Oxford University Press.

Wylie, S.A., K. Jalbert, S. Dosemagen \& M. Ratto, 2014: Institutions for Civic Technoscience. How Critical Making is Transforming Environmental Research. The Information Society 30: 116-126.

Wynne, B., 1992: Misunderstood Misunderstanding: Social Identities and Public Uptake of Science. Public Understanding of Science 1: 281-304.

Wynne, B., 2007: Public Participation in Science and Technology: Performing and Obscuring a PoliticalConceptual Category Mistake. East Asian Science, Technology and Society 1: 99-110.

\section{Autorenvorstellung}

Sascha Dickel, geb. 1978 in Gießen. Studium der Politikwissenschaft und Soziologie in Marburg und Frankfurt. Promotion in Bielefeld. Von 2009-2012 Post-Doc am Institut für Wissenschafts- und Technikforschung an der Universität Bielefeld. 2012-2014 Wissenschaftlicher Mitarbeiter am Institut für ökologische Wirtschaftsforschung in Berlin. Seit 2014 Wissenschaftlicher Mitarbeiter am Friedrich Schiedel-Lehrstuhl für Wissenschaftssoziologie an der TU München. Forschungsschwerpunkte: Gesellschaftstheorie, Wissenschafts- und Techniksoziologie, Digitalisierung, Zukunftswissen, Biopolitik.

Wichtigste Publikationen: Enhancement-Utopien. Soziologische Analysen zur Konstruktion des Neuen Menschen, Baden-Baden 2011; Die Regulierung der Zukunft. „Emerging Technologies“ und das Problem der Exklusion des Spekulativen, in: A. Bora, A. Henkel \& C. Reinhardt (Hrsg.), Wissensregulierung und Regulierungswissen, Weilerswist 2014.

Martina Franzen, geb. 1974 in Flensburg. Studium der Soziologie, Philosophie und Germanistik in Kiel und Bremen. Promotion in Bielefeld. Von 2009 bis 2014 Wissenschaftliche Mitarbeiterin an der Universität Bielefeld. Seit 2014 Wissenschaftliche Mitarbeiterin in der Forschungsgruppe Wissenschaftspolitik am Wissenschaftszentrum Berlin für Sozialforschung.

Forschungsschwerpunkte: Wissenschaftssoziologie, Medientheorie, digitaler Wandel.

Wichtigste Publikationen: Breaking News. Wissenschaftliche Zeitschriften im Kampf um Aufmerksamkeit, Baden-Baden 2011; Grenzen der wissenschaftlichen Autonomie. Zur Eigengesetzlichkeit von Publikationskulturen, in: M. Franzen, A. Jung, D. Kaldewey \& J. Korte (Hrsg.): Autonomie revisited - Beiträge zu einem umstrittenen Grundbegriff in Wissenschaft, Kunst und Politik. Sonderband 2 der Zeitschrift für Theoretische Soziologie 2014. 